\title{
Management of liver diseases during the pandemic of coronavirus disease-19
}

\author{
Ju-Yeon Cho, ${ }^{1, *}$, Soon Sun Kim ${ }^{2, *}$, Young-Sun Lee ${ }^{3}$, Do Seon Song ${ }^{4}$, Jeong-Hoon Lee ${ }^{5}$, and Ji Hoon Kim ${ }^{3}$ on \\ behalf of the Korean Association for the Study of the Liver
}

'Department of Internal Medicine, College of Medicine, Chosun University, Gwangju; '²Department of Gastroenterology, Ajou University School of Medicine, Suwon; ${ }^{3}$ Department of Internal Medicine, Guro Hospital, Korea University College of Medicine, Seoul; ${ }^{4}$ Department of Internal Medicine, St. Vincent's Hospital, The Catholic University of Korea, Seoul; ${ }^{5}$ Department of Internal Medicine and Liver Research Institute, Seoul National University College of Medicine, Seoul, Korea

Keywords: SARS-CoV-2; COVID-19; Hepatocelluar carcinoma; Liver cirrhosis; Hepatitis

\section{INTRODUCTION}

Coronavirus disease-19 (COVID-19) is a disease caused by severe acute respiratory syndrome coronavirus 2 (SARS-CoV-2). It is spreading rapidly all over the world, including Korea, which was firstly reported from Wuhan, China.' Even in this pandemonium, the treatment of patients with chronic liver disease, hepatocellular carcinoma (HCC), and management associated with liver transplantation should be elegantly continued. Precautions to be undertaken with knowledge of COVID-19 are summarized in this document.

\section{DETAILS AND RECOMMENDATIONS}

\section{Effect of SARS-CoV-2 virus on the liver}

1) SARS-CoV-2 binds to cells through angiotensin-converting enzyme 2 (ACE2). ACE2 is highly expressed in hepatocytes and bile duct epithelial cells. Thus, hepatocytes and bile duct epithelial cells can become target cells for viral infection. ${ }^{2}$ However, there is no evidence of viral inclusion within liver tissue. ${ }^{3}$

2) Liver function test (LFT) abnormalities. (1) Incidence: 14$53 \%{ }^{4-7}$ Rare cases of acute liver damage have been reported in COVID-19 patients. ${ }^{7}$ (2) Presented as: elevated serum levels of aspartate aminotransferase (AST), alanine aminotransferase (ALT), gamma glutamyl transferase, and bilirubin. LFT abnormalities can be caused by immune-cytopathic damage following direct cytotoxicity and inflammatory response of SARS-CoV-2. ${ }^{6,8}$ It is difficult to distinguish whether liver dysfunction is due to SARS-CoV-2 itself, its complications, or side effects caused by therapeutic drugs. (3) SARS-CoV-2 can influence the disease course of chronic liver disease due to its direct toxicity to the liver, reactivation of preexisting chronic hepatitis virus, and liver damage from medications targeting the SARS-CoV-2. Experimental drugs such as remdesivir,

\footnotetext{
Abbreviations:

ACE2, angiotensin-converting enzyme 2; ALT, alanine aminotransferase; ARB, angiotensin receptor blocker; AST, aspartate aminotransferase; CDC, Centers for Disease Control and Prevention; COVID-19, coronavirus disease-19; HCC, hepatocellular carcinoma; IL, interleukin; LFT, liver function test; MELD, model for end-stage liver disease; MERS, Middle East respiratory syndrome; SARS-CoV-2, severe acute respiratory syndrome coronavirus 2

*These two authors contributed equally as co-first authors.
}

\section{Corresponding author : Jeong-Hoon Lee}

Department of Internal Medicine and Liver Research Institute, Seou National University College of Medicine, 101 Daehak-ro, Jongno-gu, Seoul 03080, Korea

Tel: +82-2-2072-2228, Fax: +82-2-743-6701

E-mail: pindra@empal.com or JHLeeMD@snu.ac.kr

https://orcid.org/0000-0002-0315-2080 
chloroquine, hydroxychloroquine, tocilizumab, and statins used as therapeutic agents for COVID-19, along with antibiotics and antifungal agents used as treatments for accompanying bacterial and fungal pneumonia may induce liver toxicity. In a Chinese randomized controlled trial of remdesivir, serum bilirubin was elevated in approximately $10 \%$ of subjects in the remdesivir group, which was not significantly higher than that $(9 \%)$ in the placebo group. AST elevation was also reported in $5 \%$ of subjects in the remdesivir group, which was not significantly different from the placebo group (12\%). ${ }^{9}$ In another randomized controlled trial of remdesivir vs. placebo, the elevation of AST and ALT was not significantly more frequent in the remdesivir group than that in the placebo group. $^{10}$ (4) LFT abnormalities are more common in patients with severe COVID-19 than in patients with mild cases. ${ }^{6}$ (5) Hypoalbuminemia at hospitalization reflects the severity of COVID-19.

(6) Hepatic dysfunction is generally transient and does not require special treatment. ${ }^{6}$

3) Data on liver histology of patients with COVID-19 are limited and its characteristics are mainly nonspecific."

\section{[Considerations]}

1. If a COVID-19 patient has an abnormal liver function, serological tests including hepatitis B surface antigens and anti-hepatitis $C$ virus antibody are necessary as in the case of LFT abnormality under normal circumstances.

2. If COVID-19 is positive in patients with autoimmune hepatitis and those who have undergone liver transplantation, deterioration in LFT should not be presumed as a disease flare or an acute rejection without a biopsy confirmation.

3. To avoid infections while transporting a COVID-19 patient, cancel or postpone diagnostic tests including ultrasound, computed tomography, magnetic resonance imaging unless bile duct obstruction, cholangitis, and acute venous thrombosis are suspected.

4. Abnormality in liver function tests is not a contraindication in the use of experimental or off-label treatments for COVID-19 (remdesivir, chloroquine, hydroxychloroquine, tocilizumab, statin, etc.). However, regular monitoring of liver function tests is necessary in these patients regardless of baseline liver function test results.

5. When a patient's liver function continues to deteriorate, other causes should be considered.
A. Consider COVID-19 complications such as myositis (especially when AST exceeds ALT), ischemia, cytokine release syndrome, and drug-induced liver injury.

\section{Management of outpatients with stable liver disease}

1) Interindividual transmission of SARS-CoV-2 may occur even from asymptomatic patients to both immunocompromised and immunocompetent groups. ${ }^{12}$

2) In children, severe or fatal COVID-19 is uncommon and increases in ALT and AST are usually mild. However, transmission of the virus is still possible. ${ }^{13}$

3) About $0.6 \%$ of COVID-19 patients had chronic liver disease according to report from Centers for Disease Control and Prevention (CDC) of the USA. ${ }^{14}$ Approximately $2-11 \%$ of Chinese COVID-19 patients accompanied chronic liver disease., ${ }^{5,15}$

4) Approximately $1 \%$ of COVID-19 patients were reported to have a history of cancer. They had a higher risk of intensive care unit care or death. ${ }^{16}$ The incidence of COVID-19 or the risk of death associated with SARS-CoV-2 in HCC patients are unknown.

5) The median tumor doubling time of 4-6 months in HCC could be the rationale for a brief delay of radiological surveillance. ${ }^{17}$

\section{[Considerations]}

1. Postponing an appointment or considering a non-visit, nonface-to-face interview is necessary in the management of patients with stable liver disease.

2. Health care provider should minimize the risk of infection during patients' and accompanying guardians' hospital stay.

3. Check COVID-19-related symptoms (fever, coughing, shortness of breathing, sore throat, diarrhea, etc.) or history of travel to epidemic areas prior to appointment. Reschedule an appointment if necessary.

4. All patients who visit the hospital should be screened at the entrance for respiratory symptoms and measured body temperatures. Patients with symptoms or febrile diseases should be treated in separate screening clinics for COVID-19. They should not be permitted to hepatology clinic or liver transplant clinics.

5. Surveillance and monitoring for HCC are recommended to maintain pre-scheduled visits, but short-term delay is possible 
considering the lack of capacity in medical services amid COVID-19 pandemic according to each institution and patients' personal circumstances. (It is necessary to discuss benefits and risks caused by a delay in cancer monitoring with the patient and a documentation of the explanation is recommended.)

6. Treatment of HCCs should be continued regardless of the COVID-19 pandemic.

\section{Management of patients with decompensated cirrhosis or waiting for a liver transplant}

1) The effects of SARS-CoV-2 infection on patients with chronic liver disease, decompensated cirrhosis, and awaiting liver transplantation are not clearly understood.

2) Based on previous experience with SARS-CoV, patients with chronic liver disease (chronic hepatitis B and C) are expected to be more vulnerable to COVID-19 infection, but some studies have reported that patients with chronic liver disease do not have worse prognosis. ${ }^{18}$ Therefore, it is too early to make a conclusion with the evidence that we have so far.

3) A nationwide study in the USA showed that cirrhosis hospitalizations declined during the COVID-19 pandemic and model for end-stage liver disease (MELD) score at admission was higher in the late-COVID era compared to the previous period. ${ }^{19}$ This finding suggests that the COVID-19 pandemic delayed admission of cirrhotic patients possibly due to limited medical resources. Such indirect secondary effect of COVID-19 on cirrhotic patients needs to be considered.

\section{[Considerations]}

1. Patients with HCC or severe liver disease with high MELD scores can visit medical institutions for pre-transplant evaluation while avoiding unnecessary blood or imaging tests.

2. Educate patients not to travel during the COVID-19 pandemic.

3. Pre-transplant education should be replaced with video materials if possible. Multiple patients should not be gathered for education in a limited space. Pre-transplant counseling should also be replaced by telephone counseling.

4. Admission to a transplantation ward is strongly restricted for patients with COVID-19 symptoms.

\section{Liver transplantation and post-transplantation care}

There is no evidence that the severity of COVID-19 infection increases in patients after liver transplantation. (1) According to data reported up to date, pulmonary injury is increased due to innate immune response to SARS-CoV-2 and immunosuppression might have a protective role. ${ }^{5,20,21}$ (2) In cases of severe acute respiratory syndrome (SARS, 2003-2004) and Middle East respiratory syndrome (MERS; from 2012), immunosuppression after liver transplantation did not increase the risk of mortality. ${ }^{20}$ (3) According to a case report, a patient who was tested positive for COVID-19 immediately after liver transplantation became negative for COVID-19 after treatment but returned to positive after increasing the dosage of immunosuppressants. ${ }^{22}$

\section{[Considerations]}

1. SARS-CoV-2 positive patients are limited as candidates for organ donation.

A. Deceased donors: All deceased donors should be tested for SARS-CoV-2 before donation. They should be confirmed as negative for SARS-CoV-2 before performing an organ harvesting surgery. ${ }^{23} \mathrm{~A}$ person with the possibility of having been in contact with a patient confirmed or suspected for COVID-19 within 14 days should not be considered as a donor. Death by unknown respiratory failure should also be considered as an exclusion criterion.

B. Living organ donors and transplant recipients: Transplantation surgery for living donors and recipients who have visited an epidemic area or who are concerned about an epidemiological relevance should be deferred for 14 days of observation. For all donors and recipients, SARS-CoV-2 test result must be confirmed as negative before an operation. ${ }^{23}$

2. Medical resources (intensive care unit, ventilator, blood products, etc.) must be secured prior to transplantation.

3. Reduction of immunosuppressant or discontinuation of mycophenolate is not recommended for asymptomatic liver transplant patients unless they are COVID-19 positive.

4. The possibility of prolonged viral shedding caused by the use of immunosuppressants in transplanted patients demands attention to post-transplantation care. Examinations should be conducted in accordance with guidelines for each medical institution in case of suspected COVID-19. 


\section{Treatment of HCC patients}

1) As the COVID-19 pandemic state is prolonged, shortage of medical devices and health care providers may occur.

2) A multidisciplinary team approach is recommended to plan treatment method and appropriate time for HCC patients while considering the risk of in-hospital infection.

3) Treatment of HCC can be undertaken at the public relief hospitals.

4) HCC patients should minimize visits to hospitals in order to decrease the risk of infection. Invasive treatments should be considered for patients with low possibility of decompensation and minimal risk for developing severe COVID-19.

\section{[Considerations]}

1. There is no need to delay necessary treatment unless there is a shortage of medical devices or health care providers.

2. For patients with a high risk of developing severe COVID-19, treatment should be decided after evaluating risks and benefits.

3. Treatment response evaluation can be extended temporarily according to COVID-19 pandemic status.

4. When a patient is on a clinical trial, rearrangement of hospital visits, treatment schedule and treatment site can be considered.

5. If it is difficult to apply the first recommended treatment modality, optimal alternative therapy or intensive imaging examinations should be performed.

6. Liver transplantation can be delayed in order to protect the donor and the recipient. In such a case, the patient should be fully informed about the possibility of cancer progression and deterioration of liver function.

7. While waiting for liver transplantation, bridging therapies could be considered.

8. Visiting schedules can be rearranged in order to minimize hospital visits of patients who are on systemic therapy. Remote medical support can be considered if possible.

9. For patients receiving treatment for HCC, the risk of infection upon hospital visit should be considered.

10. If treatment continuation is not feasible, intensive imaging examinations or conservative management should be done.

11. For HCC patients who develop COVID-19, locoregional therapies should be delayed. Consideration to temporarily withdraw immune-checkpoint inhibitors should be undertaken as there is a higher risk of developing serious COVID-19 in patients receiving immunosuppressive therapy. In mild cases of COVID-19, the continuation of kinase inhibitors should be in the discretion of the clinician's judgement with each case.

\section{The use of immunosuppressants}

1) Effects of immunosuppression on the development of COVID-19 have not been established yet.

2) Rapid exacerbation of respiratory symptoms has been reported to be systemic and respiratory inflammatory reactions related to increased levels of serum interleukin (IL)-6, IL-8, and tumor necrosis factor- $a^{24,25}$

3) Potential benefits of corticosteroids in preventing the progression of symptoms in mild COVID-19 patients have not been well understood yet. However, the use of steroids in SARS and MERS patients was not advantageous in a meta-analysis. ${ }^{26}$

4) In patients with autoimmune liver disease or patients with acute rejection after liver transplantation, rapid reduction or discontinuation of immunosuppressive agents can exacerbate the course of the disease.

\section{[Considerations]}

1. Use of immunosuppressants in COVID-19-negative patients

A. djustment of the dose of immunosuppressants in advance due to the COVID-19 pandemic is not necessary.

2. Use of immunosuppressants in COVID-19-positive patients

A. Steroids or immunosuppressants can be used when potential benefits are greater than the risk.

B. Consider minimizing the dosage of high-dose steroids, but maintain a sufficient dose to avoid adrenal insufficiency or aggravation of underlying liver disease that has been controlled by steroids.

C. In cases of pneumonia aggravation, lymphopenia and persisting fever, the daily dose of azathioprine or mycophenolate can be reduced or discontinuation of the drug can be considered according to the discretion of the clinician's judgement.

D. In cases of pneumonia aggravation, lymphopenia, and persisting fever, the daily dose of calcineurin inhibitors may be reduced. However, such inhibitors should not be discontinued since calcineurin inhibitors as cornerstones 
of immunosuppression cannot be replaced by other immunosuppressants, especially in post-transplant patients.

3. Modulating immunosuppressants after liver transplantation in COVID-19 patients

A. $>6$ months from liver transplant: Consider reducing or stopping antimetabolites. Consider reducing the level of calcineurin inhibitor.

B. $<6$ months from liver transplant: Consider reducing or stopping antimetabolites. Maintain calcineurin inhibitor.

C. Early post-transplant period: Consider maintaining immunosuppressants.

\section{Inpatient care and inpatient/outpatient procedures}

1) The possibility of fecal-to-oral transmission of SARS-CoV-2 has been suggested ${ }^{1,8,27,28}$ and the virus has been identified in saliva. ${ }^{29}$

2) The stability of SARS-CoV-2 in aerosols has been confirmed, ${ }^{30}$ suggesting the possibility of virus transmission via aerosols. ${ }^{31}$

\section{[Considerations]}

1. Before admission, check travel history to prevalent areas, fever, and respiratory symptoms. Delaying hospitalization should be considered for symptomatic patients. If the emergency patient with pneumonia of unknown origin is recommended to be admitted to an isolated unit, COVID-19 infection should be tested.

2. All visitors should be checked for their travel history and symptoms such as fever, cough, and sore throat when they visit the hospital. Their access should be recorded and visits other than one guardian are strictly restricted.

3. In patients with liver disease diagnosed with COVID-19, non-emergency procedures such as elective endoscopy, liver biopsy, and etc. should be postponed as much as possible. However, urgent or emergent procedures (e.g., liver biopsy to confirm graft rejection in transplant patients, therapeutic paracentesis, transjugular intrahepatic portosystemic shunt, endoscopy for treatment of variceal bleeding, and pancreatobiliary procedures) should be performed promptly.

4. During all medical procedures, healthcare workers should comply with standard precautionary guidelines. They should wear goggles or face shields and gloves when an exposure to secretions is expected and apply KF94 or N95 masks when aerosol spread of the virus is concerned. ${ }^{32,33}$
5. After all procedures, follow appropriate disinfection guidelines for each procedure. Follow the Korean CDC instructions after performing procedures for confirmed or suspected COVID-19 patients. $^{33,34}$

\section{Medication management and potential drug-drug interactions in COVID-19 patients}

1) US Food and Drug Administration granted emergency use authorization for investigational antiviral remdesivir to treat COVID-19.

2) Remdesivir, a nucleotide analogue, is currently undergoing multiple randomized controlled studies on its effectiveness as a treatment for SARS-CoV-2 treatment. ${ }^{35,36}$ (ClinicalTrials.gov ID: NCT04292899, NCT04292730, NCT04257656, NCT04252664, and NCT04280705). (1) This drug has been demonstrated to inhibit SARS-CoV and MERS-CoV in vitro and in vivo experiments. ${ }^{37}$ (2) Data from compassionate use of remdesivir showed that $68 \%$ of severe COVID-19 patients showed clinical improvement. ${ }^{38}$ (3) The first randomized controlled trial conducted at Hubei province of China showed that remdesivir was not associated with statistically significant clinical benefits including shortened time to clinical improvement. ${ }^{39}$ However, preliminary data from another randomized controlled trial in the USA, Korea, and other countries showed that remdesivir could shorten the time to recovery in adults hospitalized with COVID-19 and evidence of lower respiratory tract infection. ${ }^{10}$

3) In randomized, open-label clinical trials comparing lopinavir/ ritonavir with conservative treatment in patients with COVID-19, there was no clinical benefit of lopinavir/ritonavir and the trial was discontinued prematurely due to adverse drug reactions. ${ }^{40}$ In addition to negative results of the clinical trial, unfavorable pharmacodynamics of lopinavir/ritonavir and reported hepatotoxicity limits its use for treating COVID-19. (1) Lopinavir/ritonavir, a potent inhibitor of CYP3A4, can affect the metabolism of calcineurin inhibitors, sirolimus, and everolimus. (2) When using lopinavir/ritonavir, the dose of tacrolimus should be reduced by $1 / 20-1 / 50$ folds

4) Tocilizumab, a drug that targets the IL-6 receptor, is being tested only in hospitalized patients with moderate to severe COVID-19. ${ }^{41}$

5) Hydroxychloroquine was confirmed to have an inhibitory effect on SARS-CoV-2 in vitro experiments. ${ }^{42}$ However, only nonrandomized studies have been conducted in clinical trials with 
conflicting results. ${ }^{43,44}$ Further studies with well-designed clinical trials are needed to confirm the antiviral effect of hydroxychloroquine against COVID-19. (1) A combination therapy with hydroxychloroquine and azithromycin did not result in clinical improvement or viral clearance and several patients presented with prolonged QT intervals. ${ }^{44}$

6) Several promising case reports of convalescent plasma transfusion have been reported in critically ill patients with COVID-19. ${ }^{45}$

7) Niclosamide, an anti-helminthic drug, exhibited antiviral properties against SARS-CoV, MERS-CoV, and more recently SARS-CoV-2. Although niclosamide suffers a pharmacokinetic flaw of low adsorption, further development of its drug formulation could enable an effective delivery of this drug to the target tissue.

8) Clevudine, a nucleoside analogue developed in Korea that can inhibit the replication of hepatitis B virus, has recently demonstrated antiviral activity against SARS-CoV-2 and further clinical research is to be initiated. ${ }^{46}$ Attention to reversible myopathy, which has been reported in treated chronic hepatitis B patients due to the depletion of mitochondrial DNA leading to mitochondrial myopathy, is warranted. ${ }^{47,48}$

9) ACE inhibitors and angiotensin receptor inhibitors (ARBs) can theoretically promote SARS-CoV-2 infection as they can increase the expression of ACE2, the target for the virus to enter cells. ${ }^{49}$ However, there has been insufficient evidence to limit ACEI/ARB treatment in COVID-19 patients because reports have shown that ACEI/ARB have cardio-pulmonary protective effects and increased expression of ACE2 can reduce acute lung injury. ${ }^{50}$

\section{[Considerations]}

1. The use of lopinavir/ritonavir for the treatment of COVID-19 is not recommended.

2. Hydroxychloroquine with or without azithromycin is not generally recommended due to the possibility of serious side effects.

3. It is recommended that patients taking ACEI/ARB maintain the drug.

\section{Healthcare workers' protection and working environment}

Health care workers and hospital staff are at risk of COVID-19 infection. ${ }^{51}$ Medical practitioners with confirmed infection of COVID-19 can spread the virus to patients.

\section{[Considerations]}

1. All medical practitioners should be aware of the latest knowledge of COVID-19 and follow infection control guidelines.

2. The hospital should secure and allocate appropriate personnel considering the level of skills and fatigue of healthcare workers.

3. Healthcare workers who anticipate to treat confirmed or suspected COVID-19 patients should always execute hand hygiene and be equipped with appropriate personal protective equipment to avoid direct contact with patient's blood, body fluids, secretions, and skin (body protective clothing or long-sleeved gowns, disposable gloves, KF94 or N95 mask, goggles, or face shield).

4. Consider replacing all in-person meetings with online video conference and follow rules of social distancing.

\section{Authors' contribution}

Manuscript preparation: Cho JY, Kim SS, Lee JH

Article reviews: Cho JY, Kim SS, Lee YS, Song DS, Lee JH, Kim JH All authors revised and approved the final version of the manuscript.

\section{Acknowledgements}

This document is approved by the Korean Association for the Study of the Liver (KASL). It is designed to provide information to clinicians on the treatment of patients with liver disease during the pandemic of COVID-19. The information provided in this document has not been subject to a heightened review to act as a standard of care or a practice guideline since new knowledge regarding the disease is continuously evolving. Management of liver diseases should be individualized according to each clinical situation and regional characteristics.

\section{Conflicts of Interest}

The authors have no conflicts to disclose.

\section{REFERENCES}

1. Guan WJ, Ni ZY, Hu Y, Liang WH, Ou CQ, He JX, et al. Clinical characteristics of coronavirus disease 2019 in China. N Engl J Med 2020;382:1708-1720.

2. Hoffmann M, Kleine-Weber $H$, Schroeder S, Krüger N, Herrler T, 
Ju-Yeon Cho, et al. Liver diseases during the COVID-19 pandemic

Erichsen S, et al. SARS-CoV-2 cell entry depends on ACE2 and TMPRSS2 and is blocked by a clinically proven protease inhibitor. Cell 2020;181:271-280.e8.

3. Wong SH, Lui RN, Sung JJ. Covid-19 and the digestive system. J Gastroenterol Hepatol 2020;35:744-748.

4. Chen N, Zhou M, Dong X, Qu J, Gong F, Han Y, et al. Epidemiological and clinical characteristics of 99 cases of 2019 novel coronavirus pneumonia in Wuhan, China: a descriptive study. Lancet 2020;395:507-513.

5. Huang C, Wang Y, Li X, Ren L, Zhao J, Hu Y, et al. Clinical features of patients infected with 2019 novel coronavirus in Wuhan, China. Lancet 2020;395:497-506.

6. Zhang C, Shi L, Wang FS. Liver injury in COVID-19: management and challenges. Lancet Gastroenterol Hepatol 2020;5:428-430.

7. Xu L, Liu J, Lu M, Yang D, Zheng X. Liver injury during highly pathogenic human coronavirus infections. Liver Int 2020;40:998-1004.

8. Gu J, Han B, Wang J. COVID-19: Gastrointestinal manifestations and potential fecal-oral transmission. Gastroenterology 2020;158: 1518-1519.

9. Wang Y, Zhang D, Du G, Du R, Zhao J, Jin Y, et al. Remdesivir in adults with severe COVID-19: a randomised, double-blind, placebocontrolled, multicentre trial. Lancet 2020;395:1569-1578.

10. Beigel JH, Tomashek KM, Dodd LE, Mehta AK, Zingman BS, Kalil AC, et al. Remdesivir for the treatment of Covid-19 - preliminary report. N Engl J Med. 2020 May 22. doi: 10.1056/NEJMoa2007764.

11. Xu Z, Shi L, Wang Y, Zhang J, Huang L, Zhang C, et al. Pathological findings of COVID-19 associated with acute respiratory distress syndrome. Lancet Respir Med 2020;8:420-422.

12. Bai $Y$, Yao L, Wei T, Tian F, Jin DY, Chen $L$, et al. Presumed asymptomatic carrier transmission of COVID-19. JAMA 2020;323:14061407.

13. Zheng F, Liao C, Fan QH, Chen HB, Zhao XG, Xie ZG, et al. Clinical characteristics of children with coronavirus disease 2019 in Hubei, China. Curr Med Sci 2020;40:275-280.

14. CDC COVID-19 Response Team. Preliminary estimates of the prevalence of selected underlying health conditions among patients with coronavirus disease 2019 - United States, February 12-March 28, 2020. MMWR Morb Mortal Wkly Rep 2020;69:382-386.

15. Xu XW, Wu XX, Jiang XG, Xu KJ, Ying LJ, Ma CL, et al. Clinical findings in a group of patients infected with the 2019 novel coronavirus (SARS-Cov-2) outside of Wuhan, China: retrospective case series. BMJ 2020;368:m606.

16. Liang W, Guan W, Chen R, Wang W, Li J, Xu K, et al. Cancer patients in SARS-CoV-2 infection: a nationwide analysis in China. Lancet Oncol 2020;21:335-337.

17. Rich NE, John BV, Parikh ND, Rowe I, Mehta N, Khatri G, et al. Hepatocellular carcinoma demonstrates heterogeneous growth patterns in a multi-center cohort of patients with cirrhosis. Hepatology.
2020 Feb 4. doi: 10.1002/hep.31159.

18. Bangash MN, Patel J, Parekh D. COVID-19 and the liver: little cause for concern. Lancet Gastroenterol Hepatol 2020;5:529-530.

19. Mahmud N, Hubbard RA, Kaplan DE, Serper M. Declining Cirrhosis hospitalizations in the wake of the COVID-19 pandemic: a national cohort study. Gastroenterology. 2020 May 5. doi: 10.1053/ j.gastro.2020.05.005.

20. D'Antiga L. Coronaviruses and immunosuppressed patients: the facts during the third epidemic. Liver Transpl 2020;26:832-834.

21. Mehta P, McAuley DF, Brown M, Sanchez E, Tattersall RS, Manson JJ, et al. COVID-19: consider cytokine storm syndromes and immunosuppression. Lancet 2020;395:1033-1034.

22. Qin J, Wang H, Qin X, Zhang P, Zhu L, Cai J, et al. Perioperative presentation of COVID-19 disease in a liver transplant recipient. Hepatology. 2020 Mar 27. doi: 10.1002/hep.31257.

23. The Korean Society for Transplantation (KST). Recommendation on COVID-19. KST web site, <http://www.mykst.org/board/ list.html? num $=700 \&$ start $=0 \&$ sort $=$ top $\% 20$ desc,reg_dt $\% 20$ desc\&code=m_notice\&key=\&keyword $=>$. Accessed 14 May 2020.

24. Li G, Fan Y, Lai Y, Han T, Li Z, Zhou P, et al. Coronavirus infections and immune responses. J Med Virol 2020;92:424-432.

25. Gong J, Dong H, Xia SQ, Huang YZ, Wang D, Zhao Y, et al. Correlation analysis between disease severity and inflammation-related parameters in patients with COVID-19 pneumonia. MedRxiv. 2020 Feb 27. doi: 10.1101/2020.02.25.20025643.

26. Russell CD, Millar JE, Baillie JK. Clinical evidence does not support corticosteroid treatment for 2019-nCoV lung injury. Lancet 2020;395:473-475.

27. Xiao F, Tang M, Zheng $X$, Liu Y, Li X, Shan $H$. Evidence for gastrointestinal infection of SARS-CoV-2. Gastroenterology 2020;158:18311833.e3.

28. Holshue ML, DeBolt C, Lindquist S, Lofy KH, Wiesman J, Bruce H, et al. First case of 2019 novel coronavirus in the United States. N Engl J Med 2020;382:929-936.

29. To KK, Tsang OT, Chik-Yan Yip C, Chan KH, Wu TC, Chan JMC, et al. Consistent detection of 2019 novel coronavirus in saliva. Clin Infect Dis. 2020 Feb 12. doi: 10.1093/cid/ciaa149.

30. van Doremalen N, Bushmaker T, Morris DH, Holbrook MG, Gamble A, Williamson BN, et al. Aerosol and surface stability of SARS-CoV-2 as compared with SARS-CoV-1. N Engl J Med 2020;382:1564-1567.

31. Wang J, Du G. COVID-19 may transmit through aerosol. Ir J Med Sci. 2020 Mar 24. doi: 10.1007/s11845-020-02218-2.

32. Korean Society of Gastrointestinal Endoscopy (KSGE). Recommendations for endoscopy units during the COVID-19 pandemic. KSGE web site, <https://www.gie.or.kr/bbs/index.html?code=notice\&c ategory $=1$ \&gubun $=\&$ page $=1 \&$ number $=5646 \&$ mode $=$ view $\&$ ord er $=\% 20$ signdate\&sort=\%20desc\&keyfield=\&key=\&page_type $=>$. Accessed 1 May 2020. 
33. Korea Centers for Disease Control and Prevention (KCDC). Prevention and management of COVID-19 in healthcare facility. KCDC web site, <http://ncov.mohw.go.kr/shBoardView.do?brdld=2\&brdGubun $=24 \&$ ncvContSeq=1277> . Accessed 1 May 2020.

34. Korea Centers for Disease Control and Prevention (KCDC). Disinfection guidelines to prevent the spread of COVID-19 at public and multi-purpose facilities. KCDC web site, <http://ncov.mohw.go.kr/ en/guidelineView.do?brdld=18\&brdGubun=181\&dataGubun=\&ncv ContSeq=2506\&contSeq=2506\&board_id=\&gubun=\#>. Accessed 1 May 2020.

35. Li G, De Clercq E. Therapeutic options for the 2019 novel coronavirus (2019-nCoV). Nat Rev Drug Discov 2020;19:149-150.

36. Wang M, Cao R, Zhang L, Yang X, Liu J, Xu M, et al. Remdesivir and chloroquine effectively inhibit the recently emerged novel coronavirus (2019-nCoV) in vitro. Cell Res 2020;30:269-271.

37. Sheahan TP, Sims AC, Graham RL, Menachery VD, Gralinski LE, Case $J B$, et al. Broad-spectrum antiviral GS-5734 inhibits both epidemic and zoonotic coronaviruses. Sci Transl Med 2017;9:eaal3653.

38. Grein J, Ohmagari N, Shin D, Diaz G, Asperges E, Castagna A, et al. Compassionate Use of remdesivir for patients with severe COVID-19. N Engl J Med 2020;382:2327-2336.

39. Wang Y, Zhang D, Du G, Du R, Zhao J, Jin Y, et al. Remdesivir in adults with severe COVID-19: a randomised, double-blind, placebocontrolled, multicentre trial. Lancet 2020;395:1569-1578.

40. Cao B, Wang Y, Wen D, Liu W, Wang J, Fan G, et al. A trial of lopinavir-ritonavir in adults hospitalized with severe COVID-19. N Engl J Med 2020;382:1787-1799.

41. Luo P, Liu Y, Qiu L, Liu X, Liu D, Li J. Tocilizumab treatment in COVID-19: a single center experience. J Med Virol 2020;92:814-818.

42. Yao $X$, Ye F, Zhang $M$, Cui $C$, Huang $B$, Niu $P$, et al. In vitro antiviral activity and projection of optimized dosing design of hydroxychloroquine for the treatment of severe acute respiratory syndrome coronavirus 2 (SARS-CoV-2). Clin Infect Dis. 2020 Mar 9. doi: 10.1093/ cid/ciaa237.
43. Gautret P, Lagier JC, Parola P, Hoang VT, Meddeb L, Mailhe M, et al. Hydroxychloroquine and azithromycin as a treatment of COVID-19: results of an open-label non-randomized clinical trial. Int J Antimicrob Agents. 2020 Mar 20. doi: 10.1016/j.jjantimicag.2020.105949.

44. Molina JM, Delaugerre C, Le Goff J, Mela-Lima B, Ponscarme D, Goldwirt $L$, et al. No evidence of rapid antiviral clearance or clinical benefit with the combination of hydroxychloroquine and azithromycin in patients with severe COVID-19 infection. Med Mal Infect 2020;50:384.

45. Shen C, Wang Z, Zhao F, Yang Y, Li J, Yuan J, et al. Treatment of 5 critically ill patients with COVID-19 with convalescent plasma. JAMA 2020;323:1582-1589.

46. Peek SF, Cote PJ, Jacob JR, Toshkov IA, Hornbuckle WE, Baldwin BH,et al. Antiviral activity of clevudine [L-FMAU, (1-(2-fluoro5-methyl-beta, L-arabinofuranosyl) uracil)] against woodchuck hepatitis virus replication and gene expression in chronically infected woodchucks (Marmota monax). Hepatology 2001;33:254-266.

47. Seok JI, Lee DK, Lee CH, Park MS, Kim SY, Kim HS, et al. Long-term therapy with clevudine for chronic hepatitis B can be associated with myopathy characterized by depletion of mitochondrial DNA. Hepatology 2009;49:2080-2086.

48. Tak WY, Park SY, Cho CM, Jung MK, Jeon SW, Kweon YO, et al. Clinical, biochemical, and pathological characteristics of clevudineassociated myopathy. J Hepatol 2010;53:261-266.

49. Fang L, Karakiulakis $G$, Roth M. Are patients with hypertension and diabetes mellitus at increased risk for COVID-19 infection? Lancet Respir Med 2020;8:e21.

50. Guo J, Huang Z, Lin L, Lv J. Coronavirus Disease 2019 (COVID-19) and cardiovascular disease: a viewpoint on the potential influence of angiotensin-converting enzyme inhibitors/angiotensin receptor blockers on onset and severity of severe acute respiratory syndrome coronavirus 2 infection. J Am Heart Assoc 2020;9:e016219.

51. Remuzzi A, Remuzzi G. COVID-19 and Italy: what next? Lancet 2020;395:1225-1228. 